\title{
TPSMA based Sensor Node Redeployment for Mobile Wireless Sensor Networks
}

\author{
H. Zainol Abidin, N.M. Din, N.A.M Radzi
}

\begin{abstract}
Randomly deployed mobile sensor nodes sometimes could not fulfill the requirements of a Wireless Sensor Network (WSN) of having a maximum coverage and minimum energy consumption. Thus, an effective mechanism is needed to ensure that the mobile sensor nodes are moved to the new positions that would be able to provide substantial maximum coverage with minimum moving distance. This paper suggests the used of Territorial Predator Scent Marking Algorithm (TPSMA) for redeployment of mobile sensor nodes. TPSMA was originally used for static sensor node placement. Two single objective approaches are studied in this paper that includes TPSMA with minimum moving distance and TPSMA with maximum coverage. The performances of the mobile WSN redeployed with the two TPSMA approaches are evaluated and compared with WSN redeployment using Genetic Algorithm (GA). Simulation results significantly show that TPSMA performs better than the GA for mobile sensor nodes redeployment.
\end{abstract}

Keywords-TPSMA, mobile, redeployment, sensor node, wireless sensor networks

\section{Introduction}

The positions of sensor nodes in a Wireless Sensor Network (WSN) affect the coverage and the energy consumption of a WSN. Proper sensor node placement should be deployed to ensure that the positions would be able to give considerable maximum coverage with lower energy consumption because these two aspects are influenced by the distance between two sensor nodes. The coverage and energy consumption are two conflicting objectives. If the two sensor nodes are placed too far, bigger coverage could be achieved but the energy consumption will be higher due to longer data transmission and vice versa [1].

Sensor node placement can be divided into two types that are known as dynamic placement and static placement. Dynamic placement may involve both homogeneous and heterogeneous sensor nodes while static placement involves homogeneous WSN where only stationary sensor nodes are

H. Zainol Abidin

Universiti Teknologi MARA

Malaysia

N.M. Din, N.A.M Radzi

Universiti Tenaga Nasional

Malaysia used. In dynamic sensor nodes placement for homogeneous WSN with mobile sensor nodes, only mobile sensor nodes are deployed randomly. These sensor nodes will move from the initial position where they were placed randomly in the monitoring area to fulfil the design objectives which is known as redeployment process.

Since most WSN can have large number of nodes, the task of selecting the positions of the sensor nodes for an optimal designed network can be very complex. Therefore, metaheuristics seem to be an interesting option to solve this problem [2]. Particle Swarm Optimization (PSO) and Genetic Algorithm (GA) based algorithms are mostly applied for sensor node placement. PSO which was introduced by Kennedy and Eberhart [3] in 1995 is a computational method that optimizes a problem by iteratively trying to improve a candidate solution with regard to a given measure of quality. It was inspired by social behaviour of bird flocking or fish schooling. PSO optimizes a problem by having a population of candidate solutions which are represented by particles that are moving around in the search-space according to simple mathematical formula over the particle's position and velocity. The movements of the particles are guided by their own best known position in the search-space as well as the entire swarm's best known position. The process is repeated until a desired solution is discovered. PSO particles converge to their own history best positions and group history best positions that will limit the search range of particles [4]. However, PSO has the disadvantage of local minima [5] and computational time coming as big constraints [6]. Some of PSO based algorithms for sensor node redeployment are Virtual Force Directed Co-Evolutionary PSO (VFCPSO) [7], traditional PSO [8], PSO with learning automata (PSO-LA) [6], Particle Swarm Frog Leaping Hybrid Optimization Algorithm [9] and Intelligent Single Particle Optimizer (ISPO) [4].

Works presented in [10-15] propose sensor node placement algorithms that are based on GA. GA is a search and optimization algorithm that mimics the process of natural selection [16]. GA belongs to the larger class of Evolutionary Algorithms (EA), which generate solutions to optimization problems using techniques inspired by natural evolution, such as inheritance, mutation, selection, and crossover. The evolution usually starts from a population of randomly generated chromosomes and it is then iteratively being processed. The population in each iteration is called a generation where in each generation, the fitness of every individual in the population is evaluated. The fitness is usually the value related to the objective function in the optimization problem to be solved. The more fit chromosomes are randomly selected from the current population. Each chromosome will go through the mutation, crossover and selection processes to form a new generation. The new generation of candidate solutions is then used in the next 
iteration of the algorithm. Commonly, the algorithm terminates when either a maximum number of generations has been produced or a satisfactory fitness level has been reached for the population. However, GA prone to premature convergence phenomenon and difficult to ensure accuracy [4] because it starts from population of random solutions and recombines and mutates them with aim to be improved without taking into account the specificity of the problem [2].

This paper presents a mobile sensor node redeployment based on Territorial Predator Scent Marking Algorithm (TPSMA) which was previously applied for static sensor node placement in [17-20]. The next section will further elaborate the TPSMA used in mobile WSN redeployment followed by our network model and problem formulation in Section III. Performance evaluation is then presented in Section IV while Section V concludes the paper.

\section{TPSMA for Sensor Node Placement}

TPSMA was inspired by the behaviour of a territorial predator in scent marking their territory. Territorial predators such as tigers and bears defend certain areas from other species based on certain factors such as food sources. Most predators scent mark a sign to show the boundaries of their territory [21]. Scent is usually marked through urination, defecation, rubbing parts of the body such as the chin and legs, scratches and destruction of vegetation, for example, tigers mark trees by spraying urine and cats rub their face and their flanks against objects. Scent matching allows animals to distinguish residents from intruders by recognizing their smell through sniffing [22]. TPSMA was first introduced in [20] for static sensor node placement. Table I tabulates the sensor node placement algorithm phases inspired by the scent marking behaviour. The territory of a sensor node can be scent marked based on a design objective such as maximum coverage, minimum uniformity, minimum energy consumption and others. This is done based on the scent marking behaviour where normally predator will scent mark the area due to certain factors such as food resources. Sensor node will be placed based on their marked territories that imitate the scent matching behaviour. Fig. 1 illustrates the flow chart of the TPSMA based sensor node placement.

TABLE I. SENSOR NODE PLACEMENT BASED ON TPSMA

\begin{tabular}{|c|c|}
\hline Sensor Node Placement & $\begin{array}{c}\text { Biological Scent Marking } \\
\text { Behaviour }\end{array}$ \\
\hline $\begin{array}{c}\text { Optimized positions of sensor nodes } \\
\text { are marked based on the design } \\
\text { objectives }\end{array}$ & $\begin{array}{c}\text { Predator marks the area } \\
\text { based on the food resources }\end{array}$ \\
\hline $\begin{array}{c}\text { Sensor nodes identify the marked } \\
\text { positions and move to the marked } \\
\text { positions }\end{array}$ & $\begin{array}{c}\text { Predator scent matching } \\
\text { behaviour through sniffing }\end{array}$ \\
\hline
\end{tabular}

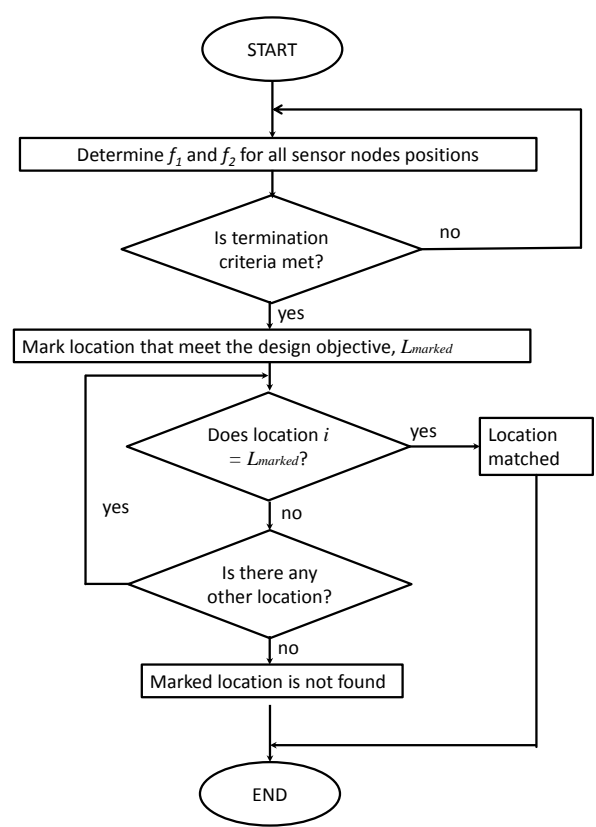

Figure 1. Flowchart of the TPSMA based sensor node placement

\section{Network Model and Problem Formulation}

A monitoring area is divided into a number of small square tiles called monitoring locations which are also the potential locations of sensor nodes. Thus, the number of monitoring locations is equal to the number of potential locations for sensor node. Each monitored location can only be equipped with one sensor node. The centre of each square tile is considered as the monitoring point and may be monitored by more than one sensor node. The area is assumed to be obstacle free. The covering area of a sensor node is assumed to be a circular area with a radius of $R_{S}$ that represents the sensing range. Monitoring points that are located within that circle is considered covered by the sensor node. At least one sensor node must be able to cover a monitoring point. Sensor nodes are homogeneous mobile sensor nodes where all sensor nodes will have similar $R_{S}$ and communication range, $R_{C}$. The number of sensor nodes must not exceed the number of monitoring locations.

There are two constraints of this optimization problem as indicated by equations (1) and (2). First, the distance between any two sensor nodes must not exceed their $R_{C}$ and there must be at least a path from the sensor node to the sink node to ensure connectivity. Only one sensor node can be placed in each monitoring location. The monitoring locations are marked with $x(p)$ as follows to indicate whether the location is equipped with a sensor node or not.

$$
x(p)=\left\{\begin{array}{lr}
1 & \text { if location } p \text { has a sensor node } \\
0 & \text { otherwise }
\end{array}\right.
$$


Proc. of the Intl. Conf. on Advances in Computing, Control and Networking - ACCN 2015.

Copyright (C) Institute of Research Engineers and Doctors, USA .All rights reserved.

ISBN: 978-1-63248-038-5 doi: 10.15224/ 978-1-63248-038-5-77

$$
\text { const }= \begin{cases}d\left(s_{i}, s_{i-1}\right) \leq R_{C}, & i \in\{1,2, \ldots, N\} \\ \alpha_{i} \geq 1, & i \in\{1,2, \ldots, N\} \\ x(p)=0, & p \in\{1,2, \ldots, M\}\end{cases}
$$

where

$N=$ number of sensor nodes

$M=$ number of monitoring points

$\alpha_{i}=$ Number of sensor nodes from sensor node $i$ to the sink node in multi-hop communication

$d\left(s_{i}, s_{i-1}\right)=$ Euclidean distance between two sensor nodes

Two single objective approaches of TPSMA for mobile sensor node redeployement are studied in this paper known as TPSMA-Moving Distance and TPSMA-Coverage.

\section{A. TPSMA-Moving Distance}

The first approach is called TPSMA-Moving Distance which is aiming at the shortest moving distance of the sensor nodes from the initial positions when it is randomly deployed to the optimized positions. The objective function of TPSMAMoving Distance is derived as shown in equations (3) and (4).

$$
\text { Movement }_{i}=d\left(s_{i I}, s_{i F}\right)
$$

where

Movement $_{i}=$ Moving distance for each sensor node

$d\left(s_{i l}, s_{i F}\right)=$ Euclidean distance between initial position of sensor node $i$ and final position of sensor node $i$

The objective function of TPSMA-Moving Distance, $f_{l}$ is the average of moving distance for each sensor node, Movement $_{\text {Ave }}$ :

$$
f_{1}=\text { Movement }_{\text {Ave }}: \frac{\sum_{i \in N} \text { Movement }_{i}}{N}
$$

\section{B. TPSMA-Coverage}

The second approach is known as TPSMA-Coverage where the objective is to move the sensor nodes to the positions that give the maximum coverage. Equations (5) and (6) derive the objective function for TPSMA-Coverage.

$$
\text { NCovered }_{p}=\left\{\begin{array}{rr}
1 & d\left(s_{i}, m_{p}\right) \leq R_{S} \\
0 & \text { otherwise }
\end{array}\right.
$$

where

NCovered $_{p}=$ Coverage for each monitoring point

$d\left(s_{i}, m_{p}\right)=$ Euclidean distance between sensor node $i$ and monitoring point $p$
The objective function of TPSMA-Coverage, $f_{2}$ is the sum of covered points, NCovered:

$$
f_{2}=\text { NCovered }=\sum_{p \in M} \text { NCovered }_{p}
$$

\section{Performance Evaluation}

The performance of the mobile WSN deployed with TPSMA is evaluated through simulation study which has been carried out by using MATLAB.

\section{A. Simulation Network Model}

Performance of the mobile WSN redeployed using TPSMA is observed in terms of the coverage ratio and the average moving distance. The coverage ratio is a ratio of number of covered monitoring points to the total monitoring points in the monitoring area. The monitoring area is considered fully covered if the ratio is 1 . The average moving distance shows the average distance travelled by each sensor node from the initial position to the optimized position. The moving distance affects the energy consumption where if the sensor node moves further, the energy consumption will also increase due to mobility energy.

A monitoring area with $60 \mathrm{~m} \times 60 \mathrm{~m}$ dimension is divided into 144 equal width monitored locations. Centre of each monitored location is called the monitoring points.

\section{B. Simulation Results}

Simulation results of mobile WSN redeployed with TPSMA sensor node placement algorithm are compared with results produced by a mobile WSN redeployed with GA because they are presenting the same network scenario and GA is also widely used for mobile WSN redeployment.

Fig. 2 depicts the coverage ratio of WSN deployed using TPSMA-Moving Distance and GA-Moving Distance. Based on the figure, it can be seen that the coverage ratio of GAMoving Distance is slightly higher compared to TPSMAMoving Distance. 22 sensor nodes are needed by TPSMAMoving Distance compared to GA-Moving Distance which needs 18 sensor nodes for full coverage. GA-Moving Distance offers higher coverage ratio because TPSMA-Moving Distance gives lower average moving distance compared to GA-Moving Distance as illustrated in Fig. 3. TPSMA offers lower moving distance because TPSMA evaluate the location from the beginning while GA starts from random solutions where they are recombined and mutated among them. Thus, the solutions provided by GA might not be the most optimum solution. Although, the TPSMA-Moving Distance gives lower coverage ratio, the difference is only around $2 \%$. On the other hand, TPSMA-Moving Distance managed to outperform the average moving distance of GA-Moving Distance on average by $11 \%$ as shown in the Fig. 3. Thus, it can be concluded that, TPSMA-Moving Distance would be able to reduce the energy consumption and hence lengthen the lifetime of the sensor nodes. 
Proc. of the Intl. Conf. on Advances in Computing, Control and Networking - ACCN 2015.

Copyright $\odot$ Institute of Research Engineers and Doctors, USA .All rights reserved.

ISBN: 978-1-63248-038-5 doi: 10.15224/ 978-1-63248-038-5-77

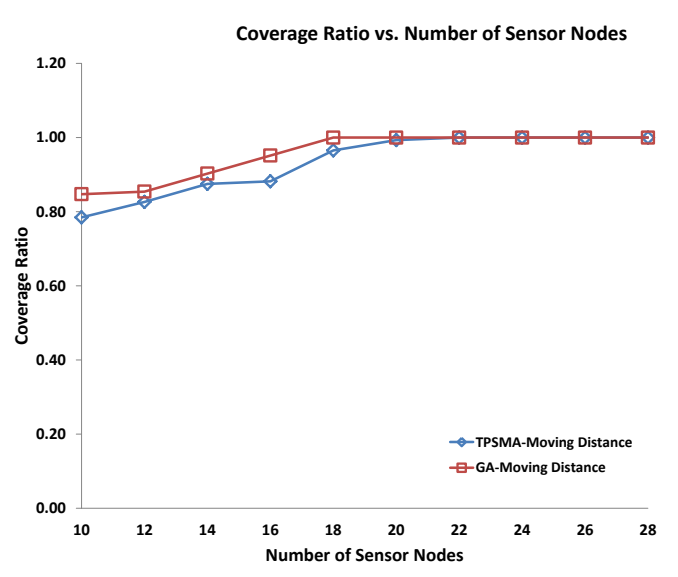

Figure 2. Coverage ratio of TPSMA-Moving Distance and GA-Moving Distance.

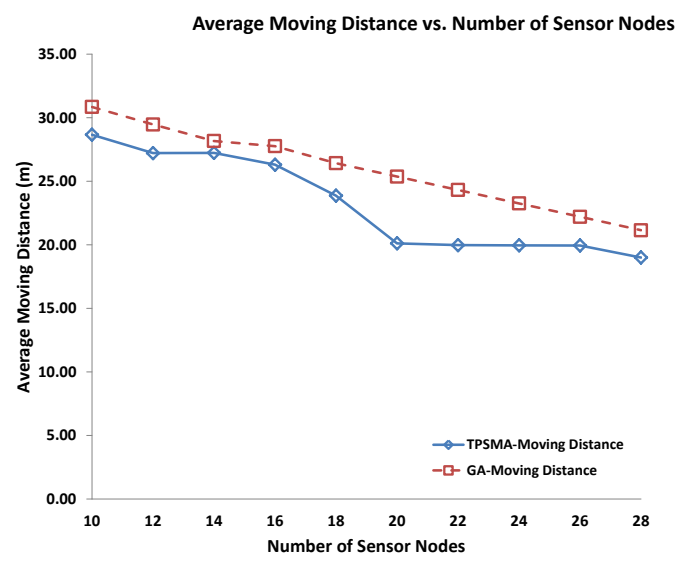

Figure 3. Average moving distance of TPSMA-Moving Distance and GAMoving Distance.

Fig. 4 exhibits the coverage ratio of WSN deployed using TPSMA and GA with maximum coverage ratio objective function. It can be seen that the coverage ratio produced by TPSMA-Coverage is higher than GA-Coverage. With 10 sensor nodes, TPSMA-Coverage is able to provide 0.96 coverage ratio compared to GA-Coverage which is able to cover 0.90 of the area. TPSMA-Coverage offers full coverage by deploying 14 sensor nodes compared to GA-Coverage which needs 18 sensor nodes. Thus, the deployment cost for full coverage WSN can be reduced by $22 \%$ compared to GACoverage. In short, it can be said that the deployment of TPSMA is considered cost effective even though the main objective is to provide maximum coverage. TPSMA offers higher coverage because TPSMA evaluate the location that will give maximum coverage from the beginning while GA starts from random solutions where they are recombined and mutated among them. Thus, the solutions provided by GA might not be the most optimum solution.
Average moving distance of mobile sensor nodes in a WSN deployed with TPSMA-Coverage and GA-Coverage is presented in Fig. 5. The trend of the graph shows that the average moving distance of the sensor nodes are decreasing as the number of sensor nodes increase. This is because as the number of sensor nodes increase, the sensor nodes does not need to move further because there are more sensor nodes to cover the monitoring area. As can be seen, the average moving distance of each mobile sensor node of WSN deployed with TPSMA-Coverage is higher compared to GA-Coverage. On average there is a difference around $13 \%$ between these two algorithms. TPSMA-Coverage moved further compared to GA-Coverage because it offers higher coverage ratio as shown in the Fig. 4. This is because, sensor nodes will need to move further in order to maximize the coverage area. It is proven now that there is a trade-off between the coverage of the WSN and the moving distance of the sensor nodes.



Figure 4. Coverage ratio of TPSMA-Coverage and GA-Coverage.

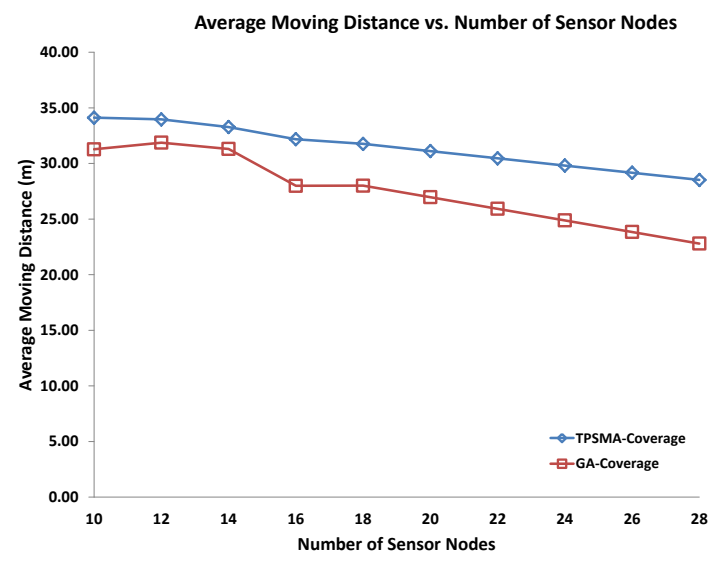

Figure 5. Average moving distance of TPSMA-Coverage and GA-Coverage. 


\section{Proc. of the Intl. Conf. on Advances in Computing, Control and Networking - ACCN 2015.}

Copyright (C) Institute of Research Engineers and Doctors, USA .All rights reserved.

ISBN: 978-1-63248-038-5 doi: 10.15224/ 978-1-63248-038-5-77

\section{v. Conclusion}

TPSMA was previously used for static sensor node placement. In this paper, TPSMA is used for mobile sensor node redeployment where the mobile sensor nodes will move to the positions determined by the TPSMA. Two single objective TPSMA approaches were applied that are known as TPSMA-Moving Distance and TPSMA-Coverage. The performances of both TPSMA approaches are compared with GA. Based on the simulation results, it can be seen that TPSMA- Moving Distance have the shorter average moving distance compared to GA-Moving Distance. However, the coverage ratio is smaller due to shorter moving distance. TPSMA-Coverage offers bigger coverage compared to GACoverage consequently the average moving distance is higher in order to accommodate bigger coverage. From the simulation results, it can be concluded that, TPSMA would be able to give better solutions compared to GA. This is proven where TPSMA-Moving Distance had a shorter movement compared to GA-Moving Distance while TPSMA-Coverage provide bigger coverage compared to GA-Coverage. It can also be seen that there is a trade-off between the coverage and moving distance where if the coverage is bigger, the moving distance will become longer. Thus, a multi objective approach could be developed in order to solve the trade-off between the coverage and moving distance.

\section{References}

[1] P. M. Pradhan and G. Panda, "Connectivity constrained wireless sensor deployment using multiobjective evolutionary algorithms and fuzzy decision making," Ad Hoc Networks, vol. 10, pp. 1134-1145, 2012.

[2] S. Fidanova, P. Marinov, and E. Alba, "Ant Algorithm for optimal sensor deployment," Computational Intelligence, vol. 399, pp. 21-29, 2012.

[3] J. Kennedy and R. Eberhart, "Particle swarm optimization," in IEEE International Conference on Neural Networks, 1995, pp. 1942-1948.

[4] J. Zhao and H. Sun, "Intelligent single particle optimizer based wireless sensor networks adaptive coverage," Journal of Convergence Information Technology, vol. 7, pp. 153-159, 2012.

[5] W. Xue, M. Jun-Jie, W. Sheng, and B. Dao-Wei, "Distributed particle swarm optimization and simulated annealing for energy-efficient coverage in wireless sensor networks," Sensors, vol. 7, pp. 628-648, 2007.

[6] R. Soleimanzadeh, B. J. Farahani, and M. Fathy, "PSO based deployment algorithms in hybrid sensor networks," International Journal of Computer Science and Network Security, vol. 10, pp. 167-171, 2010.

[7] X. Wang and S. Wang, "Hierarchical deployment optimization for wireless sensor networks," IEEE Transactions on Mobile Computing, vol. 10, pp. 1028-1041, 2010.

[8] N. Kukunuru, B. R. Thella, and R. L. Davuluri, "Sensor deployment using particle swarm optimization," International Journal of Engineering Science and Technology, vol. 2, pp. 5395-5401, 2010.

[9] H. Sun and J. Zhao, "Application of particle sharing based particle swarm frog leaping hybrid optimization algorithm in wireless sensor network coverage optimization," Journal of Information and Computational Science, vol. 8, pp. 3181-3188, 2011.

[10] Y. Yoon and Y.-H. Kim, "An efficient genetic algorithm for maximum coverage deployment in wireless sensor networks," IEEE Transactions on Cybernetics, vol. 43, pp. 1473-1483, 2013.

[11] L. Zhang, D. Li, H. Zhu, and L. Cui, "OPEN: An optimisation scheme of N-node coverage in wireless sensor networks," Wireless Sensor Systems, IET, vol. 2, pp. 40-51, 2012.
[12] V. V. Juli and J. Raja, "Mobility assisted optimization algorithms for sensor node deployment," European Journal of Scientific Research, vol. 78, pp. 156-167, 2012.

[13] T. E. Kalayci and A. Ugur, "Genetic algorithm-based sensor deployment with area priority," Cybernetics and Systems, vol. 42, pp. 605-620, 2011.

[14] N. Rahmani and F. Nematy, "EAVD: An evolutionary approach diagram for node deployment in wireless sensor networks," Advances in Intelligent and Soft Computing vol. 130, pp. 121-129 2012.

[15] N. Rahmani, F. Nematy, A. M. Rahmani, and M. Hosseinzadeh, "Node placement for maximum coverage based on voronoi diagram using genetic algorithm in wireless sensor networks," Australian Journal of Basic and Applied Sciences, vol. 5, pp. 3221-3232, 2011.

[16] R. L. Haupt and S. E. Haupt, Practical Genetic Algorithms. New Jersey: John Wiley and Sons, 2004.

[17] H. Z. Abidin and N. M. Din, "Energy efficient sensor nodes placement using Territorial Predator Scent Marking Algorithm (TPSMA)," in 4th International Conference on Energy and Environment 2013 (ICEE 2013), IOP Conf. Series: Earth and Environmental Science 16, Putrajaya, 2013.

[18] H. Z. Abidin and N. M. Din, "WSN Sensor Node Placement Approach using Territorial Predator Scent Marking Algorithm (TPSMA) " in 2013 IEEE 11th Malaysia International Conference on Communication (MICC 2013), Kuala Lumpur, 2013.

[19] H. Z. Abidin, N. M. Din, and N. A. M. Radzi, "Deterministic Static Sensor Node Placement in Wireless Sensor Network based on Territorial Predator Scent Marking Behaviour," Journal of Communication Networks and Information Security (IJCNIS), vol. 5, pp. 186 - 191, 2013

[20] H. Zainol Abidin and N. M. Din, "Sensor Node Placement in Wireless Sensor Network Based on Territorial Predator Scent Marking Algorithm," ISRN Sensor Networks, vol. 2013, p. 7, 2013.

[21] C. M. Begg, K. S. Begg, J. T. Du Toit, and M. G. L. Mills, "Scentmarking behaviour of the honey badger, Mellivora capensis (Mustelidae), in the southern Kalahari," Animal Behaviour, vol. 66, pp. 917-929, 2003

[22] K. A. Descovich, A. T. Lisle, S. Johnston, V. Nicolson, and C. J. C. Phillips, "Differential responses of captive southern hairy-nosed wombats (Lasiorhinus latifrons) to the presence of faeces from different species and male and female conspecifics," Applied Animal Behaviour Science, vol. 138, pp. 110-117, 2012.

About Authors:

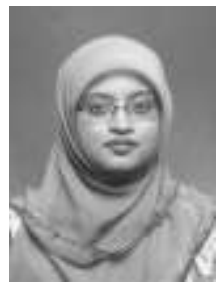

Husna Zainol Abidin received her B.Eng in Electrical from the University of Wollongong, Australia in 2001 and the M.Eng in Electrical from the Universiti Tenaga Nasional, Malaysia in 2006. She is currently working as a Senior Lecturer in the Universiti Teknologi MARA, Malaysia and a PhD in Engineering candidate in the Universiti Tenaga Nasional, Malaysia. Her main research areas of interest are data communication and wireless networking. She is a member of IEEE.

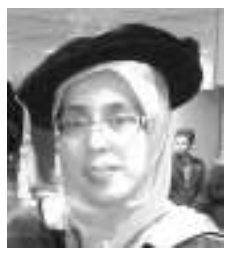

Norashidah Md Din is currently working as a Professor and Dean at College of Graduate Studies, Universiti Tenaga Nasional, Malaysia. She has a degree in Electrical Engineering from Memphis State University, USA and a Master and $\mathrm{PhD}$ degrees in Electrical Engineering from Universiti Teknologi Malaysia. She has authored over 100 technical papers. She is a Senior Member of IEEE, a CEng with IET and PEng with Board of Engineers Malaysia. Her research interests include communications network and Ethernet passive optical networks. 
Proc. of the Intl. Conf. on Advances in Computing, Control and Networking - ACCN 2015.

Copyright $\odot$ Institute of Research Engineers and Doctors, USA .All rights reserved.

ISBN: 978-1-63248-038-5 doi: 10.15224/ 978-1-63248-038-5-77

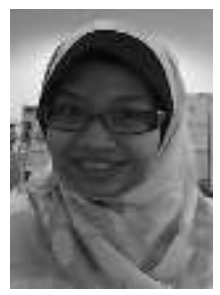

Nurul Asyikin Mohd. Radzi received her $\mathrm{PhD}$. in Engineering, MEE and BEEE (Hons.) from Universiti Tenaga Nasional in the year 2013, 2010 and 2008, respectively. In 2009, she joined the Department of Electronics and Communication Engineering, Universiti Tenaga Nasional as a tutor. She is currently working as a senior lecturer in the same department. Her research interests include, EPON and IP Optical. She has contributed 30 technical papers in various journals and conference. She is a member of IEEE. Since 2008, she has completed 4 sponsored projects. 